\title{
Incidence, trends and demographics of Staphylococcus aureus infections in Auckland, New Zealand, 2001-2011
}

Deborah A Williamson 1,2,3,5*, Alwin Lim ${ }^{1}$, Mark G Thomas ${ }^{1}$, Michael G Baker ${ }^{4}$, Sally A Roberts' ${ }^{2}$, John D Fraser ${ }^{1}$ and Stephen R Ritchie ${ }^{1}$

\begin{abstract}
Background: New Zealand has a higher incidence of Staphylococcus aureus disease than other developed countries, with significant sociodemographic variation in incidence rates. In contrast to North America, the majority of disease is due to methicillin-susceptible S. aureus (MSSA), although relatively little is known about the comparative demographics of MSSA and methicillin-resistant S. aureus (MRSA) infections in New Zealand.

Methods: Our objectives were to describe the trends, incidence and patient demographics of all S. aureus infections in patients presenting to our institution between 2001 and 2011, and compare the epidemiology of MSSA and MRSA infections. We identified all patients with $S$. aureus infections over the study period. A unique S. aureus infection was defined as the first positive $S$. aureus culture taken from the same patient within a thirty-day period. Standard definitions were used to classify episodes into community- or healthcare-associated S. aureus infection.

Results: There were 16,249 S. aureus infections over the study period. The incidence increased significantly over the study period from 360 to 412 per 100,000 population $(P<0.001)$, largely driven by an increase in community-associated non-invasive MSSA infections. When compared with MSSA infections, patients with non-multiresistant MRSA infections were more likely to be older, have hospital-onset infections and be Māori or Pacific Peoples.

Conclusions: Our work provides valuable baseline data on the epidemiology and trends of S. aureus infections in New Zealand. The significant increase in community-associated S. aureus infections is of public health importance. Future studies should investigate the reasons underlying this concerning trend.
\end{abstract}

Keywords: Staphylococcus aureus, Epidemiology, Healthcare-associated infection, Ethnicity, Methicillin-susceptible

\section{Background}

Staphylococcus aureus is a major human pathogen, and infections caused by $S$. aureus result in significant morbidity and mortality [1,2]. Although most commonly associated with skin and soft tissue infections (SSTI), $S$. aureus is also responsible for a range of serious invasive infections, including osteomyelitis, necrotizing pneumonia and bacteremia [3]. In addition, S. aureus disease occurs commonly in both community and healthcare settings amongst a variety of demographic groups $[1,4,5]$. To date,

\footnotetext{
* Correspondence: deb.williamson@auckland.ac.nz

${ }^{1}$ Faculty of Medical and Health Sciences, University of Auckland, Auckland, New Zealand

${ }^{2}$ Department of Clinical Microbiology, Auckland District Health Board, Auckland, New Zealand

Full list of author information is available at the end of the article
}

many studies assessing the burden of $S$. aureus infections have focused on one particular aspect of $S$. aureus disease, such as a specific clinical syndrome (e.g. bloodstream infections or SSTI), specific patients (e.g. adults only) or a specific place of acquisition (e.g. healthcare-associated infections) [6-8]. Furthermore, as a consequence of the recent epidemic of community-associated methicillin resistant S. aureus (CA-MRSA) in North America, many studies have focused exclusively on describing the epidemiology and burden of MRSA infections $[9,10]$. As such, population-based data on the overall extent of $S$. aureus disease, regardless of clinical syndrome, healthcare exposure or antimicrobial resistance profile, are limited.

The reported incidence of $S$. aureus disease in New Zealand is higher than that from similar developed

\section{Biomed Central}


countries [11-13]. However, in contrast to several other settings, particularly North America, the vast majority of $S$. aureus disease in New Zealand is due to methicillinsusceptible S. aureus (MSSA) [13-15]. Distinct demographic associations have been described for S. aureus infections in New Zealand, including age, ethnicity and economic deprivation $[11,16,17]$. To date however, relatively little is known about the comparative epidemiology of infections caused by MSSA and MRSA strains in our setting. Moreover, no studies have systematically assessed longitudinal trends in the overall incidence and epidemiology of S. aureus infections in New Zealand. Accordingly, we sought to: (i) describe the incidence, trends and patient characteristics of $S$. aureus infections in patients presenting to our hospital over an 11-year period, and (ii) compare the demographics of infections caused by MSSA and MRSA strains.

\section{Methods}

\section{Setting, patients and isolates}

Auckland District Health Board (ADHB) is a tertiarylevel, university-affiliated institution exclusively serving a population of approximately 500,000, within a larger metropolitan region of 1.4 million inhabitants. Auckland is the largest city in New Zealand, and has an ethnically diverse population, having the following major population groups: European (52\%); Asian (29\%), Pacific Peoples (11\%); Māori (indigenous New Zealander, 8\%) and other ethnicities (2\%) [18].

All specimens that cultured $S$. aureus between January $1^{\text {st }} 2001$ and December $31^{\text {st }} 2011$ were identified from the laboratory database in the Department of Clinical Microbiology, Auckland City Hospital, New Zealand. In order to restrict the analysis to isolates that were likely to represent clinically relevant infections, all screening isolates (anterior nares; axillae; groin) and isolates from genital swabs, pharyngeal swabs and stool were excluded. In addition, all specimens that did not have a culture site specified were excluded. A sterile culture site was defined as one matching the US Centers for Disease Control and Prevention (CDC) surveillance systems definition [8]. A unique S. aureus infection was defined as the first positive $S$. aureus culture taken from the same patient within a thirty-day period. All $S$. aureus isolates were identified using standardised laboratory protocols. Antimicrobial susceptibility testing was performed by agar dilution and results were interpreted according to Clinical and Laboratory Standards Institute recommendations [19]. In keeping with previous studies, strains that were phenotypically resistant to $<3$ non $\beta$-lactam antimicrobials were defined as non-multidrugresistant MRSA (nmMRSA), and strains that were resistant to $\geq 3$ non- $\beta$-lactam antimicrobials were defined as multidrug-resistant MRSA (mMRSA) [20,21].

\section{Data collection}

The following demographic information was extracted from the hospital administrative database about each patient who had an inpatient hospital admission associated with a positive $S$. aureus culture: age, gender, ethnicity, residence in a long term care facility, and number of previous hospitalizations in the preceding twelve months. In addition, hospital discharge diagnoses related to each hospital admission (coded using the International Classification of Diseases, Tenth Edition, [ICD-10] codes) were obtained for each patient. The NZ Deprivation index (NZDep) score was used to assign socioeconomic status to each patient. This score is an area-based measure of deprivation derived from New Zealand census data, and is based on various measures of deprivation, including: household income, household ownership, household occupancy, employment and education levels, and access to telecommunications [22]. It is expressed as a score between one and ten, a score of ten representing the most deprived neighbourhoods.

Population denominator information for the ADHB catchment region was obtained from the 2001 and 2006 New Zealand censuses, and from linear interpolation between census data for the study period [18]. For analysis, ethnicity was grouped into four major groupings: European, Mãori, Pacific Peoples and Other ethnicities.

\section{Definitions of Staphylococcus aureus-related hospital admissions}

Based on previously described methodology, a list of ICD-10 codes for S. aureus-related clinical syndromes were developed (a detailed list of codes are shown in Additional file 1) [23-25]. These syndromes were: (i) skin and soft tissue infection (SSTI); (ii) musculoskeletal infection; (iii) respiratory infection; (iv) endovascular infection; (v) central nervous system infection, and (vi) sepsis/bacteremia. For analysis, these categories were further classified as either non-invasive infections (SSTI) or invasive infections (all other clinical syndromes). All S. aureus bloodstream infection were classified as invasive infections. In addition, other invasive S. aureus infections were defined by the isolation of $S$. aureus from a clinical specimen, plus $\geq 1 I C D-10$ discharge diagnoses associated with an invasive clinical syndrome (Additional file 1). For positive $S$. aureus culture results with $\geq 1$ ICD-10 discharge codes associated with an invasive clinical syndrome, the principal discharge code was regarded as the most likely clinical syndrome. A non-invasive infection was defined as the isolation of $S$. aureus from a non-sterile site, with a principal or secondary $I C D-10$ discharge diagnosis associated with a SSTI (Additional file 1) and no ICD-10 codes associated with an invasive clinical syndrome.

Based on definitions adapted from similar studies, $[17,26]$ cases were described as community-associated if 
S. aureus was isolated from a patient within 48 hours of hospital admission who: (i) had no history of hospitalization or surgery in the preceding calendar year, (ii) did not reside in a long-term care facility (LTCF), and (iii) did not have any prior or current ICD-10 discharge diagnoses relating to hemodialysis. Conversely, cases were described as healthcare-associated (HCA) $S$. aureus infection if one or more of these risk factors were documented. Healthcare-associated cases were further classified as hospital-onset (HCA-HO) or communityonset (HCA-CO) depending on whether the specimen was taken $>48$ hours or $\leq 48$ hours respectively, following hospital admission.

\section{Statistical analysis}

Categorical variables were compared using the $x^{2}$ or Fisher's exact test as appropriate. Non-parametric data were compared using the Mann-Whitney $U$ test or Kruskal-Wallis test. Incidence rates were calculated per 100,000 population, and a Poisson log-linear regression model was used to assess trends in incidence rates using $\log$ population denominator data as the offset variable. Multivariate logistic regression analysis using a stepwise backward elimination model was used to identify factors associated with nmMRSA infections. All statistical analysis was performed using GraphPad Prism (Version 5.02) or STATA (Version 11) and a two-tailed $P$ value of $<0.05$ was considered significant.

\section{Ethics}

Auckland District Health Board, New Zealand, granted institutional approval for this study.

\section{Results}

\section{Patients and incidence rates}

Over the 11-year study period there were 26,244 unique S. aureus isolates identified from the laboratory database, including 16,249 patients with $\geq 1 \quad I C D$-10 discharge diagnoses consistent with a $S$. aureus-related clinical syndrome. Of these, 3,752/16,249 (23\%) patients were
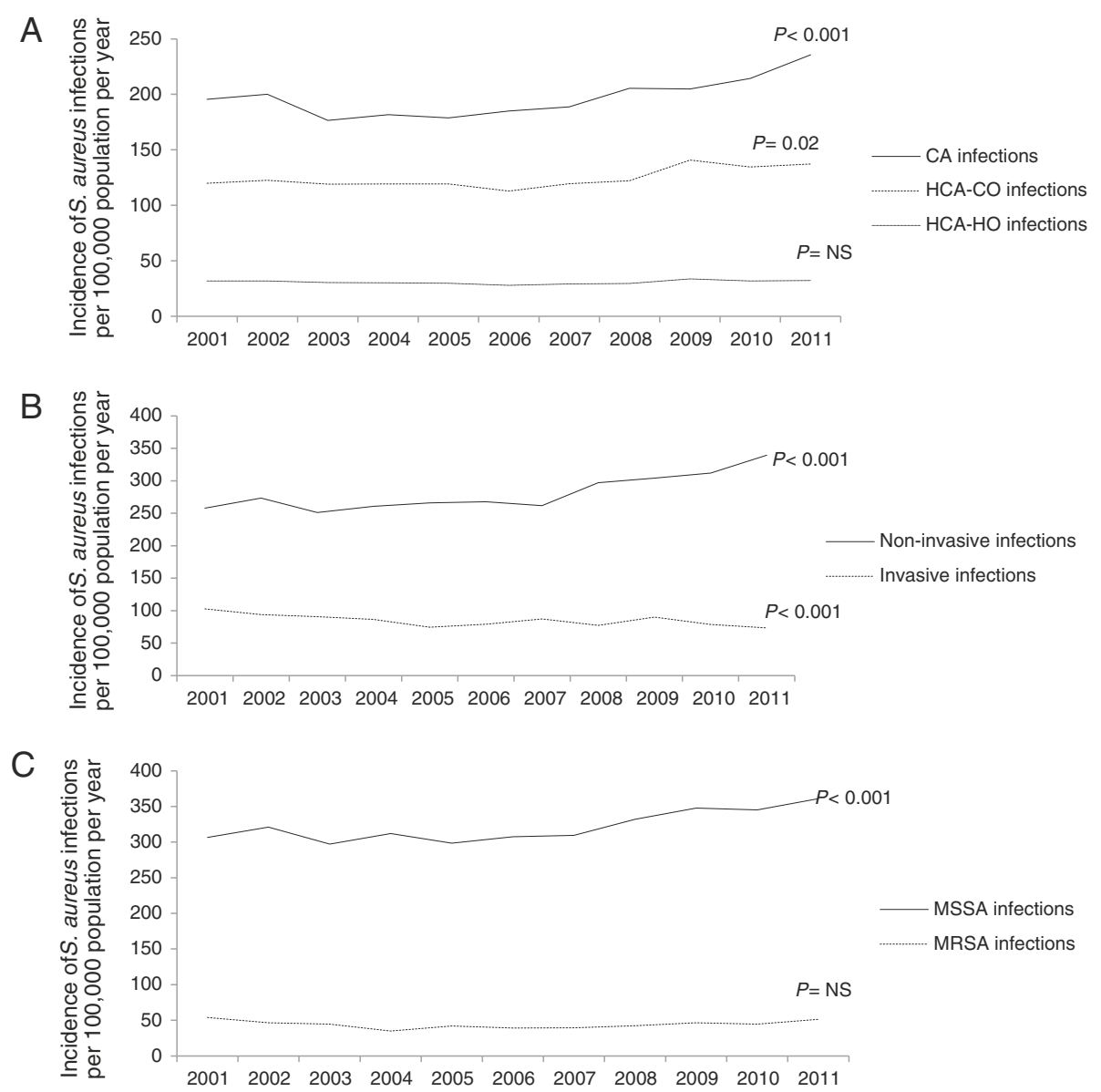

Figure 1 Incidence of Staphylococcus aureus infections per 100,000 population per year in patients hospitalized at Auckland District Health Board, New Zealand, 2001-2011, stratified by (A) place of acquisition; (B) invasive vs. non-invasive infection and (C) MSSA vs. MRSA. Abbreviations: CA, community-associated; HCA-CO, healthcare-associated, community-onset; HCA-HO, healthcare-associated, hospital-onset; MSSA, methicillin-susceptible Staphylococcus aureus; MRSA, methicillin-resistant Staphylococcus aureus; NS, not significant. 

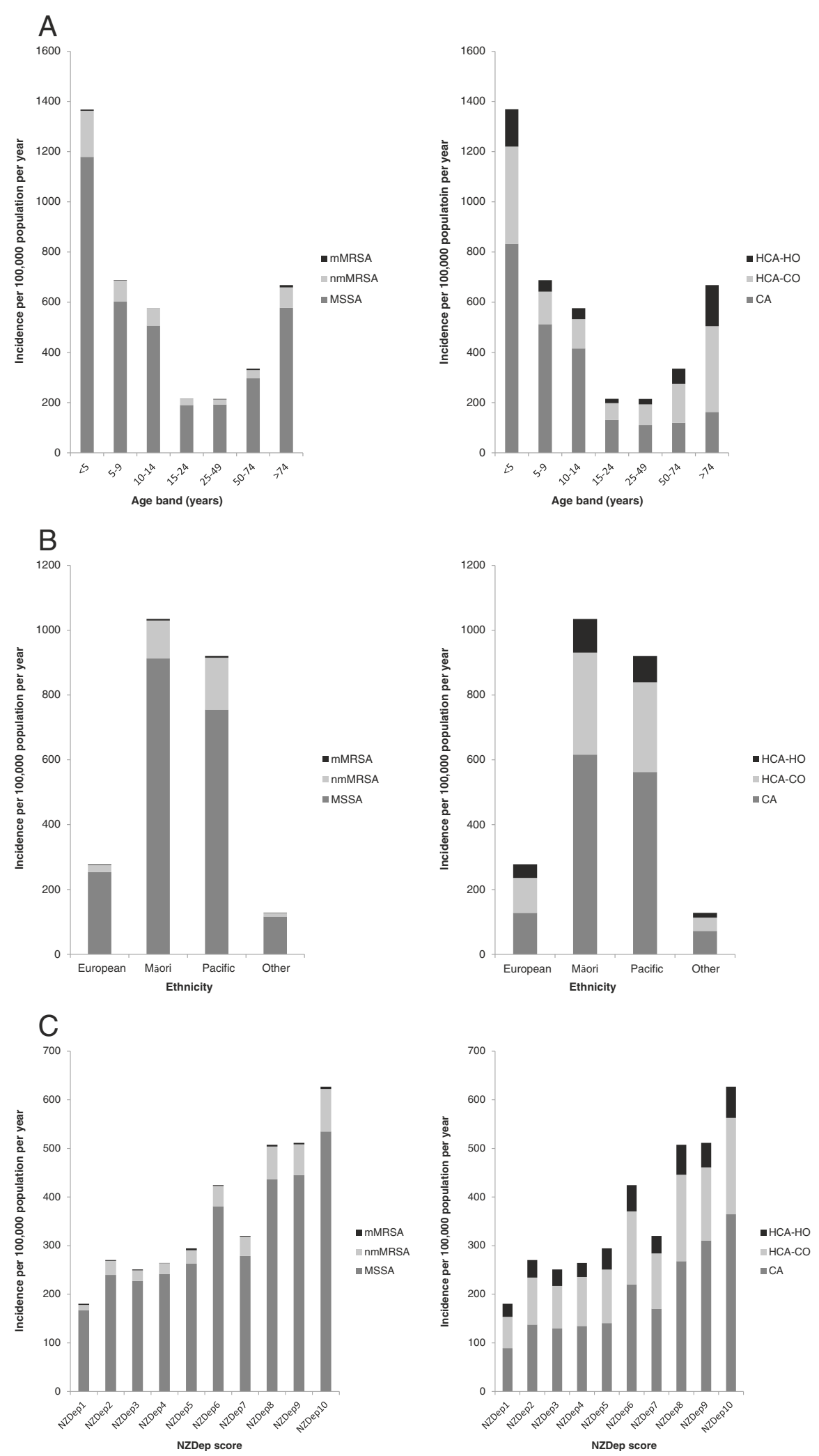

Figure $\mathbf{2}$ (See legend on next page.) 
(See figure on previous page.)

Figure 2 Incidence of Staphylococcus aureus infections per 100,000 population per year in patients hospitalized at Auckland District Health Board, New Zealand, 2001-2011, stratified by (A) age; (B) ethnicity; and (C) NZDep score according to (i) place of acquisition and (ii) MSSA, nmMRSA and mMRSA. Abbreviations: CA, community-associated; HCA-CO, healthcare-associated, community-onset; $\mathrm{HCA}-\mathrm{HO}$, healthcare-associated, hospital-onset; MSSA, methicillin-susceptible Staphylococcus aureus; MRSA, methicillin-resistant Staphylococcus aureus; NZDep, New Zealand Deprivation Index ( 1 = least deprived, 10 = most deprived).

classified as having an invasive infection, and 12,497/ $16,249(77 \%)$ as having a non-invasive infection. Overall, $8,754 / 16,249(54 \%)$ of infections were classified as community-associated, 5,523/16,249 (34\%) as HCA-CO, and 1,972/16,249 (12\%) as HCA-HO (Figure 1). The 11-year averaged incidence of all $S$. aureus relatedhospitalizations over the study period was 366 per 100,000 population per year, and increased significantly from 360 per 100,000 population in 2001, to 412 per 100,000 population in $2011(P<0.001)$. This rise was related to significant increases in community-associated $(P<0.001)$ and HCA-CO $(P=0.02)$ infections (Figure 1A); non-invasive $(P<0.001)$ infections (Figure $1 \mathrm{~B})$; and those caused by MSSA strains $(P<0.001)$ (Figure $1 C)$. The incidence of invasive $S$. aureus infections decreased significantly over the study period from 102 to 73 per 100,000 population per year $(P<0.001)$ (Figure $1 \mathrm{~B})$, and there was no significant change in the incidence of HCA-HO infections (Figure 1A). When stratified by age (Figure 2A), the incidence of $S$. aureus-related hospitalizations was highest in the under five and over 75 year age groups (1305 and 646 per 100,000 population per year respectively), and when stratified by ethnicity (Figure 2B), the incidence was highest in Māori and Pacific Peoples (981 and 873 per 100,000 population per year respectively). In addition, when stratified by NZDep score (Figure 2C), the highest incidence of $S$. aureus-related hospitalizations was in those patients residing in areas of high socioeconomic deprivation (higher NZDep scores) (Figure 2C).

\section{Characteristics of Staphylococcus aureus infections} in relation to place of acquisition and clinical syndrome The median age of all patients with $S$. aureus infections was 22 years (interquartile range [IQR] 6-50). There was a statistically significant gradient in the age distribution of patients according to place of acquisition, with the median age highest in patients with HCA-HO infections (median age 40 years, IQR 8-67), and lowest in patients with community-associated infections (median age 13 years, IQR 4-37) (Table 1). The proportion of

Table 1 Characteristics and patient demographics of Staphylococcus aureus infections, Auckland District Health Board, New Zealand, 2001-2011

\begin{tabular}{|c|c|c|c|c|c|}
\hline Characteristic & $\begin{array}{c}\text { All S. aureus } \\
\text { infections } \\
(n=16,249)\end{array}$ & $\begin{array}{l}\text { Community- } \\
\text { onset (CO) } \\
(n=8,754) \\
\end{array}$ & $\begin{array}{l}\text { Healthcare-associated, } \\
\text { community-onset } \\
\text { (HCA-CO) }(n=5,523)\end{array}$ & $\begin{array}{c}\text { Healthcare-associated, } \\
\text { hospital-onset } \\
\text { (HCA-HO) }(n=1,972)\end{array}$ & $P$ value \\
\hline Age, median, years (IQR) & $22(6-50)$ & $13(4-37)$ & $35(10-60)$ & $40(8-67)$ & $<0.001^{c}$ \\
\hline Gender, male & $9,098(56)$ & $4,967(57)$ & 2,965 (54) & $1,166(59)$ & $<0.001^{d}$ \\
\hline \multicolumn{6}{|l|}{ Clinical syndrome } \\
\hline Invasive & $3,752(23)$ & $1,220(14)$ & $1,427(26)$ & $1,105(56)$ & $<0.001^{\mathrm{d}}$ \\
\hline Non-invasive & $12,497(77)$ & $7,534(86)$ & 4,096 (74) & $867(44)$ & - \\
\hline \multicolumn{6}{|l|}{ Ethnicity $^{a}$} \\
\hline European & $6,824(42)$ & $3,114(36)$ & $2,658(48)$ & $1,052(54)$ & $<0.001^{\mathrm{d}}$ \\
\hline Māori & $3,211(20)$ & $1,908(22)$ & $978(18)$ & $325(17)$ & $<0.001^{\mathrm{d}}$ \\
\hline Pacific peoples & $4,802(30)$ & $2,932(34)$ & $1,444(26)$ & $426(22)$ & $<0.001^{\mathrm{d}}$ \\
\hline Other & $1,327(8)$ & $743(8)$ & $427(8)$ & $157(8)$ & $0.25^{\mathrm{d}}$ \\
\hline \multicolumn{6}{|l|}{ NZDep ${ }^{b}$} \\
\hline $1-3$ & $3,093(20)$ & $1,553(19)$ & $1,105(21)$ & $435(23)$ & $<0.001^{d}$ \\
\hline $4-7$ & $5,443(35)$ & $2,780(34)$ & $1,976(37)$ & $687(37)$ & \\
\hline \multirow[t]{2}{*}{$8-10$} & $6,861(45)$ & $3,915(47)$ & 2,202 (42) & $744(40)$ & $0.015^{d}$ \\
\hline & & & & & $<0.001^{d}$ \\
\hline
\end{tabular}

${ }^{\mathrm{a}}$ Data available for 16,164 infections (8,697 CO; 5,507 HCA-CO; 1,960 HCA-HO).

${ }^{\mathrm{b}}$ Data available for 15,397 infections (8,248 CO; 5,283 HCA-CO; 1,866 HCA-HO).

${ }^{\mathrm{c}} P$ value calculated using Kruskal-Wallis test.

${ }^{\mathrm{d}} P$ value calculated using $\mathrm{X}^{2}$ test.

Abbreviations: IQR interquartile range, MSSA methicillin-susceptible Staphylococcus aureus, MRSA methicillin-resistant Staphylococcus aureus, CO community-onset,

$H C A-C O$ healthcare-associated, community-onset, $H C A-H O$ healthcare-associated, hospital-onset. 
patients with invasive infections differed significantly according to place of acquisition, with $56 \%$ of HCA-HO infections classified as invasive, compared to only $26 \%$ of HCA-CO and $14 \%$ of community-associated infections $(P<0.001)$. Patients of European ethnicity were more likely to have HCA-HO infections than communityassociated infections ( $54 \%$ vs. $36 \%$; $P<0.001$ ), whereas Māori or Pacific patients were more likely to have community-associated than HCA-HO infections (22\% vs. $17 \% ; P<0.001$ and $34 \%$ vs. $22 \% ; P<0.001$ respectively) (Table 1). In addition, there was variation in the socioeconomic distribution of $S$. aureus infections across acquisition category, such that patients residing in less deprived areas were more likely to have HCA-HO infections than community-associated infections ( $23 \%$ vs. $19 \%$; $P<0.001)$. Conversely, patients in more deprived areas were more likely to have community-associated infections than HCA-HO infections ( $47 \%$ vs. $40 \%$; $P<0.001$ ) (Table 1 ).

\section{Comparison of MSSA, nmMRSA and mMRSA infections}

The incidence of MSSA infections increased significantly from 306 per 100,000 in 2001 to 361 per 100,000 in
$2011(P<0.001)$. In contrast, the incidence of MRSA did not increase over the study period (Figure 1). Overall, $1,957 / 16,249$ (12\%) of infections were caused by MRSA (1,804 [94\%] nmMRSA and 117 [6\%] mMRSA). There were distinct demographic differences between patients with MSSA, nmMRSA and mMRSA infections, such that patients with mMRSA infections were significantly older than patients with either MSSA (median 52 years vs. 22 years; $P<0.001$ ) or nmMRSA (median 52 years vs. 16 years; $P<0.001$ ), and were more likely to have invasive infections than patients with MSSA (44\% vs. $24 \% ; P<0.001)$ or nmMRSA $(44 \%$ vs. $17 \% ; P<0.001)$. Patients with MSSA or nmMRSA did not differ significantly according to the place of acquisition of their infection (Table 2). However, when compared to patients with nmMRSA, patients with mMRSA infections were significantly more likely to have HCA-CO or HCA-HO infections (50\% vs. $33 \% ; P<0.001$ and $25 \%$ vs. $13 \%$; $P<0.001$, respectively).

Given that the number of patients with mMRSA infections was relatively small $(\mathrm{n}=117)$ compared to patients with MSSA ( $\mathrm{n}=14,292)$ or nmMRSA $(\mathrm{n}=1,840)$, we

Table 2 Patient characteristics associated with methicillin-susceptible Staphylococcus aureus (MSSA) infections, non-multidrug resistant methicillin resistant S. aureus (nmMRSA) infections and multidrug resistant MRSA (mMRSA) infections, Auckland District Health Board, New Zealand, 2001-2011

\begin{tabular}{|c|c|c|c|c|c|c|c|}
\hline Characteristic & $\begin{array}{c}\text { MSSA } \\
(n=14,292)\end{array}$ & $\begin{array}{c}\text { nmMRSA } \\
(n=1840)\end{array}$ & $\begin{array}{c}\text { mMRSA } \\
(n=117)\end{array}$ & $P$ & $\begin{array}{c}\text { OR of MSSA vs. } \\
\text { nmMRSA }(95 \% \mathrm{Cl})\end{array}$ & $P$ & $\begin{array}{c}\text { OR nmMRSA vs. } \\
\text { mMRSA infection }(95 \% \mathrm{Cl})\end{array}$ \\
\hline Age, median, IQR ${ }^{a}$ & $22(6-50)$ & $16(3-47)$ & $52(31.5-71.5)$ & $<0.001$ & - & $<0.001$ & - \\
\hline Male sex ${ }^{b}$ & $8,028(56)$ & $1,005(55)$ & $69(59)$ & 0.34 & $1.19(0.83-1.69)$ & 0.23 & $0.85-1.04$ \\
\hline \multicolumn{8}{|l|}{ Clinical syndrome $^{b}$} \\
\hline Invasive & $3,390(24)$ & $311(17)$ & $51(44)$ & $<0.001$ & $1.53(1.35-1.74)$ & $<0.001$ & $0.26(0.18-0.39)$ \\
\hline Non-invasive & $10,902(76)$ & $1,529(83)$ & $66(56)$ & - & - & - & - \\
\hline \multicolumn{8}{|l|}{ Place of acquisition ${ }^{b}$} \\
\hline $\mathrm{CO}$ & $7,732(54)$ & $993(54)$ & $29(25)$ & 0.93 & $1.0(0.91-1.11)$ & $<0.001$ & $3.56(2.32-5.47)$ \\
\hline $\mathrm{HCA}-\mathrm{CO}$ & $4,851(34)$ & $614(33)$ & $58(50)$ & 0.64 & $1.03(0.93-1.14)$ & $<0.001$ & $0.51(0.35-0.74)$ \\
\hline $\mathrm{HCA}-\mathrm{HO}$ & $1,709(12)$ & $233(13)$ & $30(25)$ & 0.40 & $0.94(0.81-1.08)$ & $<0.001$ & $0.42(0.27-0.65)$ \\
\hline \multicolumn{8}{|l|}{ Ethnicity $^{\mathrm{b}, \mathrm{c}}$} \\
\hline European & $6,223(44)$ & $544(30)$ & $57(49)$ & $<0.001$ & $1.84(1.65-2.04)$ & $<0.001$ & $0.44(0.30-0.64)$ \\
\hline Māori & $2,845(20)$ & 349 (19) & $17(15)$ & 0.37 & 1.06 (0.94-1.19) & 0.27 & $1.37(0.81-2.33)$ \\
\hline Pacific Peoples & $3,942(28)$ & $832(46)$ & $28(24)$ & $<0.001$ & $0.41(0.37-0.46)$ & $<0.001$ & $2.92(1.89-4.51)$ \\
\hline Other & $1,210(8)$ & $103(5)$ & $14(12)$ & $<0.001$ & $1.56(1.27-1.92)$ & 0.013 & $0.44(0.24-0.79)$ \\
\hline \multicolumn{8}{|l|}{ NZDep band ${ }^{\text {b,d }}$} \\
\hline $1-3$ & $2,792(21)$ & $275(16)$ & $26(25)$ & $<0.001$ & $1.37(1.20-1.57)$ & 0.029 & $0.58(0.37-0.92)$ \\
\hline $4-7$ & $4,864(36)$ & $546(32)$ & $33(31)$ & $<0.001$ & 1.21 (1.09-1.35) & 1.0 & $1.02(0.67-1.56)$ \\
\hline $8-10$ & $5,903(44)$ & $911(53)$ & $47(44)$ & $<0.001$ & $0.69(0.63-0.77)$ & 0.11 & $1.39(0.94-2.07)$ \\
\hline
\end{tabular}

${ }^{a} P$ value calculated using Kruskal Wallis test.

${ }^{\mathrm{b}} P$ value calculated using $\mathrm{X}^{2}$ test.

'Data available for 16,164 infections (14,220 MSSA, 1,828 nmMRSA and 116 mMRSA).

${ }^{\mathrm{d} D a t a}$ available for 15,397 infections (13,559 MSSA, 1,732 nmMRSA and 106 mMRSA).

Abbreviations: $O R$ odds ratio, $C l$ confidence interval, IQR interquartile range, MSSA methicillin-susceptible Staphylococcus aureus, nmMRSA non-multidrug resistant methicillin-resistant Staphylococcus aureus, mMRSA multidrug resistant methicillin-resistant Staphylococcus aureus, CO community-onset, $H C A-C O$ healthcare-associated, community-onset, HCA-HO healthcare-associated, hospital-onset. 
concentrated on comparing risk factors associated with either MSSA or nmMRSA infections (Table 3). On multivariate analysis, factors associated with nmMRSA isolation were: age over 75 years (odds ratio (OR), 1.77 [95\% CI, 1.44-2.17]); Māori or Pacific ethnicity (OR, 1.48 [95\% CI, 1.26-1.75] and OR, 2.41 [95\% CI, 2.09-2.78] respectively); and HCA-HO infection (OR, 1.37 [95\% CI, 1.15-1.62]). In addition, nmMRSA infections were less likely to be invasive than MSSA infections (OR 0.64 [95\% CI, 0.55-0.74]).

\section{Discussion}

In this study, we systematically assessed the trends, incidence and demographics of patients hospitalized with S. aureus infections in our setting. We found notable demographic differences between community and healthcare-

\section{Table 3 Multivariate analysis of factors associated with non-multidrug resistant methicillin-resistant Staphylococcus aureus (nmMRSA) infections, Auckland District Health Board, New Zealand, 2001-2011}

\begin{tabular}{|c|c|}
\hline Characteristic & OR of nmMRSA vs. MSSA infection $(95 \% \mathrm{Cl})$ \\
\hline \multicolumn{2}{|l|}{ Sex } \\
\hline Male & Reference \\
\hline Female & $0.96(0.86-1.06)$ \\
\hline \multicolumn{2}{|l|}{ Type of infection } \\
\hline Non-invasive & Reference \\
\hline Invasive & $0.64(0.55-0.74)$ \\
\hline \multicolumn{2}{|l|}{ Age band (years) } \\
\hline 15 to 49 & Reference \\
\hline Under 5 & $0.98(0.85-1.14)$ \\
\hline 5 to 14 & $1.12(0.97-1.29)$ \\
\hline 50 to 74 & $1.09(0.92-1.08)$ \\
\hline Over 75 & $1.77(1.44-2.17)$ \\
\hline \multicolumn{2}{|l|}{ Ethnicity } \\
\hline European & Reference \\
\hline Māori & $1.48(1.26-1.75)$ \\
\hline Pacific peoples & $2.41(2.09-2.78)$ \\
\hline Other & $0.99(0.79-1.25)$ \\
\hline \multicolumn{2}{|l|}{ NZDep score } \\
\hline $1-3$ (low) & Reference \\
\hline 4-7 (medium) & $0.98(0.84-1.15)$ \\
\hline 8-10 (high) & $1.10(1.14-1.29)$ \\
\hline \multicolumn{2}{|c|}{ Place of acquisition } \\
\hline $\mathrm{CO}$ & Reference \\
\hline $\mathrm{HCA}-\mathrm{CO}$ & $1.08(0.96-1.21)$ \\
\hline $\mathrm{HCA}-\mathrm{HO}$ & $1.37(1.15-1.62)$ \\
\hline
\end{tabular}

Abbreviations: $O R$ odds ratio, $C l$ confidence interval, MSSA methicillin-susceptible Staphylococcus aureus, nmMRSA non-multidrug resistant methicillin-resistant Staphylococcus aureus, CO community-onset, HCA-CO healthcare-associated, community-onset, $H C A-H O$ healthcare-associated, hospital-onset. associated $S$. aureus infections, as well as differences in the comparative epidemiology of MSSA and MRSA. Our findings have implications for the following reasons.

First, by assessing all $S$. aureus infections, rather than just one specific aspect such as MRSA or nosocomial infections, our data provide a broad and comprehensive representation of the trends and burden of $S$. aureus disease in our setting. We observed an increase in the overall incidence of $S$. aureus infection, largely driven by an increase in community-associated non-invasive infections. Our finding of a significant increase in the incidence of hospitalizations for skin infections is in keeping with other recent studies in New Zealand [11,23,24], and further highlights this concerning national trend. Although reasons for this increase are unclear, suggested contributory factors include delayed access to healthcare, increases in household crowding and declining socioeconomic circumstances for specific population groups $[23,24]$. However, in contrast to the increase in noninvasive infections, the incidence of invasive $S$. aureus infections decreased significantly over the study period from 102 to 73 per 100,000 population per year $(P<0.001)$. This trend is in keeping with recent reports from North America, $[10,27,28]$ and may, in part, be due to improved local infection control practices, such as improvements in hand hygiene compliance and measures to reduce healthcare-associated bloodstream infections, including those caused by S. aureus [29].

Second, we found that in our setting, the vast majority $(88 \%)$ of S. aureus infections were caused by MSSA, and the incidence of MSSA increased significantly over the study period. Conversely, the incidence of MRSA infections remained stable; therefore the percentage contribution of MRSA to the overall burden of $S$. aureus disease actually decreased. This finding is in sharp contrast to North America, where studies suggest approximately $40-60 \%$ of $S$. aureus infections are caused by MRSA, largely due to the epidemic spread of the USA300 clone $[10,28]$. However, despite the large burden of MSSA infections in our setting, relatively little is known about the molecular epidemiology of MSSA strains in New Zealand. In particular, the extent to which the observed increase in community-associated MSSA SSTI is driven by the spread of one MSSA clone, or is due to a range of MSSA lineages is unknown. Interestingly, after multivariate analysis, we found that nmMRSA infections were more common than MSSA infections in Māori and Pacific Peoples compared to European patients. The reasons for this observation are unclear, although numerous studies have described an association between nmMRSA isolation and other distinct ethnic groups, for example African-Americans [28] and Australian Aboriginals [21]. Moreover, an association between specific communityassociated MRSA clones and Indigenous ethnic groups 
has recently been demonstrated in New Zealand [17]. Future work should investigate the possible host, bacterial and socioenvironmental factors that predispose such groups to infection with certain MRSA clones.

In our locale, patients with multiresistant MRSA represented a distinct epidemiological group, in that they were older, more likely to be European and more likely to have healthcare-associated infections than patients with MSSA or nmMRSA. This finding is consistent with a recent study from our setting, which showed that specific multiresistant MRSA clones (e.g. sequence type (ST) 22 MRSA and ST239 MRSA) were associated with prior healthcare exposure, and were more likely to be isolated from older European patients [17]. It is possible that our observation may reflect circulation and acquisition of mMRSA in long-term residential care facilities, with elderly European patients markedly overrepresented in long-term residential care facilities in New Zealand compared to non-European and Indigenous groups [30].

There were several limitations with our study. In particular, our analysis was restricted to patients with S. aureus-related hospitalizations, and did not include patients who only received treatment in primary care. Furthermore, we used a relatively conservative and specific method of case ascertainment in that we only included patients who had culture-proven $S$. aureus disease plus a hospital discharge code describing a $S$. aureus-related clinical syndrome, rather than all patients who had a positive $S$. aureus culture. Moreover, most patients discharged from hospital with an infectious disease diagnosis do not have any identified etiological agent [24]. As such, the true burden of S. aureus disease in our setting is likely to be considerably higher. In addition, we did not have patient-level information on factors such as medical co-morbidities or antibiotic usage. However, our main aim was not to provide detailed descriptive information on specific clinical syndromes, but rather to provide a broad overview of the demographics and trends of a large number of $S$. aureus infections.

\section{Conclusions}

In conclusion, our study provides valuable baseline information on the epidemiology and trends of S. aureus infections in a New Zealand population. There were notable sociodemographic differences in disease burden, with the incidence highest in Māori and Pacific Peoples. Importantly, we observed a steady and significant increase in $S$. aureus infections, predominantly due to a rise in non-invasive community-associated MSSA. Future work should investigate the clinical and molecular epidemiological associations underlying this concerning trend.

\section{Additional file}

Additional file 1: International Classification of Diseases, Tenth Revision (ICD-10) codes for Staphylococcus aureus-related infections and associated clinical syndromes.

\section{Competing interests}

The authors declare that they have no competing interests.

\section{Authors' contributions}

DAW conceived the study, participated in data collection and analysis, and drafted the manuscript. AL participated in data collection and analysis. MGT, MGB, SAR and SRR provided intellectual contributions to the manuscript sufficient to justify authorship. All authors read and approved the final manuscript.

\section{Acknowledgements}

D.A.W. is supported by a Clinical Research Training Fellowship from the Health Research Council of New Zealand. All authors report no conflict of interest.

\section{Author details}

${ }^{1}$ Faculty of Medical and Health Sciences, University of Auckland, Auckland, New Zealand. ${ }^{2}$ Department of Clinical Microbiology, Auckland District Health Board, Auckland, New Zealand. ${ }^{3}$ Institute of Environmental Science and Research, Wellington, New Zealand. ${ }^{4}$ Department of Public Health, University of Otago, Wellington, New Zealand. ${ }^{5}$ Mailing address: Department of Molecular Medicine and Pathology, University of Auckland, Private Bag 92019, Auckland, New Zealand.

Received: 3 September 2013 Accepted: 18 November 2013 Published: 3 December 2013

\section{References}

1. Laupland KB, Lyytikainen O, Sogaard M, Kennedy KJ, Knudsen JD, Ostergaard C, Galbraith JC, Valiquette L, Jacobsson G, Collignon P, Schonheyder HC, for the International Bacteremia Surveillance Collaborative: The changing epidemiology of Staphylococcus aureus bloodstream infection: a multinational population-based surveillance study. Clin Microbiol Infect 2013, 19:465-471.

2. Uslan DZ, Crane SJ, Steckelberg JM, Cockerill FR III, St Sauver JL, Wilson WR, Baddour LM: Age- and sex-associated trends in bloodstream infection: a population-based study in Olmsted County, Minnesota. Arch Intern Med 2007, 167:834-839.

3. Boucher HW, Corey GR: Epidemiology of methicillin-resistant Staphylococcus aureus. Clin Infect Dis 2008, 46(Suppl 5):S344-S349.

4. Laupland KB, Church DL, Mucenski M, Sutherland LR, Davies HD: Populationbased study of the epidemiology of and the risk factors for invasive Staphylococcus aureus infections. J Infect Dis 2003, 187:1452-1459.

5. Tong SY, van Hal SJ, Einsiedel L, Currie BJ, Turnidge JD: Impact of ethnicity and socio-economic status on Staphylococcus aureus bacteremia incidence and mortality: a heavy burden in indigenous Australians. BMC Infect Dis 2012, 12:249.

6. Turnidge JD, Kotsanas D, Munckhof W, Roberts S, Bennett CM, Nimmo GR, Coombs GW, Murray RJ, Howden B, Johnson PD, Dowling K, Australia New Zealand Cooperative on Outcomes in Staphylococcal Sepsis: Staphylococcus aureus bacteraemia: a major cause of mortality in Australia and New Zealand. Med J Aust 2009, 191:368-373.

7. Laupland KB, Ross T, Gregson DB: Staphylococcus aureus bloodstream infections: risk factors, outcomes, and the influence of methicillin resistance in Calgary, Canada, 2000-2006. J Infect Dis 2008, 198:336-343.

8. Klevens RM, Morrison MA, Nadle J, Petit S, Gershman K, Ray S, Harrison LH, Lynfield R, Dumyati G, Townes JM, Craig AS, Zell ER, Fosheim GE, McDougal LK, Carey RB, Fridkin SK, Active Bacterial Core surveillance (ABCS) MRSA Investigators: Invasive methicillin-resistant Staphylococcus aureus infections in the United States. JAMA 2007, 298:1763-1771.

9. Farr AM, Aden B, Weiss D, Nash D, Marx MA: Trends in hospitalization for community-associated methicillin-resistant Staphylococcus aureus in New York City, 1997-2006: data from New York State's Statewide Planning and Research Cooperative System. Infect Control Hosp Epidemiol 2012, 33:725-731. 
10. Hadler JL, Petit S, Mandour M, Cartter ML: Trends in invasive infection with methicillin-resistant Staphylococcus aureus, Connecticut, USA, 2001-2010. Emerg Infect Dis 2012, 18:917-924

11. Williamson DA, Ritchie SR, Lennon D, Roberts SA, Stewart J, Thomas MG Baker MG: Increasing incidence and sociodemographic variation in community-onset Staphylococcus aureus skin and soft tissue infections in New Zealand Children. Pediatr Infect Dis J 2013, 32:923-925.

12. el Atrouni WI, Knoll BM, Lahr BD, Eckel-Passow JE, Sia IG, Baddour LM: Temporal trends in the incidence of Staphylococcus aureus bacteremia in Olmsted County, Minnesota, 1998 to 2005: a population-based study. Clin Infect Dis 2009, 49(12):e130-e138.

13. Hill PC, Birch M, Chambers S, Drinkovic D, Ellis-Pegler RB, Everts R, Murdoch D, Pottumarthy S, Roberts SA, Swager C, Taylor SL, Thomas MG, Wong CG, Morris AJ: Prospective study of 424 cases of Staphylococcus aureus bacteraemia: determination of factors affecting incidence and mortality. Intern Med J 2001, 31:97-103

14. Muttaiyah S, Coombs G, Pandey S, Reed P, Ritchie S, Lennon D, Roberts S: Incidence, risk factors, and outcomes of Panton-Valentine leukocidinpositive methicillin-susceptible Staphylococcus aureus infections in Auckland, New Zealand. J Clin Microbiol 2010, 48:3470-3474.

15. Hill PC, Wong CG, Voss LM, Taylor SL, Pottumarthy S, Drinkovic D, Morris AJ: Prospective study of 125 cases of Staphylococcus aureus bacteremia in children in New Zealand. Pediatr Infect Dis J 2001, 20:868-873.

16. Ritchie SR, Fraser JD, Libby E, Morris AJ, Rainey PB, Thomas MG: Demographic variation in community-based MRSA skin and soft tissue infection in Auckland, New Zealand. N Z Med J 2011, 124:21-30.

17. Williamson DA, Roberts SA, Ritchie SR, Coombs GW, Fraser JD, Heffernan $\mathrm{H}$ : Clinical and molecular epidemiology of methicillin-resistant Staphylococcus aureus in New Zealand: Rapid emergence of Sequence Type 5 (ST5)-SCCmec-IV as the Dominant Community-Associated MRSA Clone. PLoS One 2013, 8(4):e62020.

18. Statistics New Zealand: 2006 census of populations and dwellings. Available: http://www.stats.govt.nz. Accessed 24 ${ }^{\text {th }}$ August, 2013.

19. Clinical and Laboratory Standards Institute: Performance standards forantimicrobial susceptibility testing; twenty-first informational supplement, Document M100-S22. Wayne, PA: CLSI; 2012

20. Munckhof WJ, Nimmo GR, Carney J, Schooneveldt JM, Huygens F, Inman-Bamber J, Tong E, Morton A, Giffard P: Methicillin-susceptible, non-multiresistant methicillin-resistant and multiresistant methicillin-resistant Staphylococcus aureus infections: a clinical, epidemiological and microbiological comparative study. Eur J Clin Microbiol Infect Dis 2008, 27(5):355-364.

21. Tong SY, Bishop EJ, Lilliebridge RA, Cheng AC, Spasova-Penkova Z, Holt DC, Giffard PM, McDonald MI, Currie BJ, Boutlis CS: Community-associated strains of methicillin-resistant Staphylococcus aureus and methicillinsusceptible S. aureus in indigenous Northern Australia: epidemiology and outcomes. J Infect Dis 2009, 199:1461-1470.

22. Salmond C, Crampton P, Sutton F: NZDep91: A New Zealand index of deprivation. Aust N Z J Public Health 1998, 22:835-837.

23. O'Sullivan CE, Baker MG, Zhang J: Increasing hospitalizations for serious skin infections in New Zealand children, 1990-2007. Epidemiol Infect 2011, 139:1794-1804.

24. Baker MG, Barnard LT, Kvalsvig A, Verrall A, Zhang J, Keall M, Wilson N, Wall T, Howden-Chapman P: Increasing incidence of serious infectious diseases and inequalities in New Zealand: a national epidemiological study. Lancet 2012, 379:1112-1119.

25. Tracy LA, Furuno JP, Harris AD, Singer M, Langenberg P, Roghmann MC: Predictive ability of positive clinical culture results and International Classification of Diseases, Ninth Revision, to identify and classify noninvasive Staphylococcus aureus infections: a validation study. Infect Control Hosp Epidemiol 2010, 31:694-700.

26. Williamson DA, Lim A, Roberts SA, Wiles S, Freeman J: Population-based incidence and comparative demographics of community-associated and healthcare-associated Escherichia coli bloodstream infection in Auckland, New Zealand, 2005-2011. BMC Infect Dis 2013, 3(1):385.

27. Kallen AJ, Mu Y, Bulens S, Reingold A, Petit S, Gershman K, Ray SM, Harrison LH, Lynfield R, Dumyati G, Townes JM, Schaffner W, Patel PR, Fridkin SK, Active Bacterial Core surveillance (ABCS) MRSA Investigators of the Emerging Infections Program: Health care-associated invasive MRSA infections, 2005-2008. JAMA 2010, 304:641-648.
28. Ray GT, Suaya JA, Baxter R: Trends and characteristics of cultureconfirmed Staphylococcus aureus infections in a large U.S. integrated health care organization. J Clin Microbio/ 2012, 50(6):1950-1957.

29. Roberts SA, Sieczkowski C, Campbell T, Balla G, Keenan A, Auckland District Health Board Hand Hygiene Steering and Working Groups: Implementing and sustaining a hand hygiene culture change programme at Auckland District Health Board. N Z Med J 2012, 125:75-85.

30. Kiata L, Kerse N, Dixon R: Residential care workers and residents: the New Zealand story. N Z Med J 2005, 118:U1445.

doi:10.1186/1471-2334-13-569

Cite this article as: Williamson et al:: Incidence, trends and demographics of Staphylococcus aureus infections in Auckland, New Zealand, 2001-2011. BMC Infectious Diseases 2013 13:569.

\section{Submit your next manuscript to BioMed Central and take full advantage of:}

- Convenient online submission

- Thorough peer review

- No space constraints or color figure charges

- Immediate publication on acceptance

- Inclusion in PubMed, CAS, Scopus and Google Scholar

- Research which is freely available for redistribution

Submit your manuscript at www.biomedcentral.com/submit
C) BioMed Central 